Ohnishi, Masao

Osaka Math. J.

13 (1961), 125-137.

\title{
Gentzen Decision Procedures for Lewis's Systems S2 and S3
}

\author{
By Masao OHNISHI
}

In [5] $\mathrm{K}$. Matsumoto and the author gave a Gentzen decision procedure for Lewis's system $S 2 .{ }^{1)}$ But the solution was an indirect one: we formulated a sequential calculus $Q 2$ (indentical with E2*, see below), and proving a cut-elimination theorem for it we gave a Gentzen decision procedure for $Q 2$, then we established the reduction of the decision problem of S2 to that of Q2. In [4] Matsumoto gave a Gentzen decision procedure for $S 3^{1)}$; the solution was indirect in just the same sense as mentioned above.

In this paper we shall first present two sequential calculi $S 2^{*}$ and $S 3^{*}$ equivalent to $S 2$ and $S 3$ respectively, then we shall prove cutelimination theorems for them. Therefore direct solutions of Gentzen type will be given for both systems S2 and S3.

The author expresses his hearty thanks to Mr. Matsumoto for many helpful comments on an earlier draft of this paper.

\section{$\$ 1$. Formulations of $S 2$ and $S 2^{*}$.}

Our consideration of Lewis's system $S 2$ will be based upon the formulation of E. J. Lemmon [2], which consists of full classical propositional calculus with the following two additional axioms:

$$
\begin{gathered}
(p-q)>(\square p \supset \square q)^{2)} ; \\
\square p \supset p ;
\end{gathered}
$$

and the following two additional rules of inference:

$\left(\mathrm{a}^{\prime}\right)$ if $\alpha$ is a tautology or an axiom, then $\square \alpha$ is provable ;

(b) if $\alpha-3 \beta$ is provable, then so is $\square \alpha-3 \square \beta$.

Now we shall present a sequential calculus $S 2^{*}$, which consists of all propositional rules of inference of Gentzen's $L K$ and the following two rules of modality:

1) Lewis and Langford [3].

2) As logical symbols we adopt \& (and), $\vee$ (or), $\sim$ (not), $\supset$ (material implication) and $\square$ (necessity). $\alpha-3 \beta$ and $\square(\alpha \supset \beta)$ are identical. We refer to axioms and rules by Lemmon's designations. 


$$
\left\{\begin{array}{l}
\frac{\alpha, \Gamma \rightarrow \Theta}{\square \alpha, \Gamma \rightarrow \Theta}(\square \rightarrow) ; \\
\frac{\Gamma \rightarrow \alpha}{\square \Gamma \rightarrow \square \alpha}(\rightarrow \square) ;
\end{array}\right.
$$

where $\Gamma, \Theta$ etc. mean (void or non-void) series of formulas, and $\square \Gamma$ means the series which arises when we prefix $\square$ to each formula belonging to $\Gamma$. In case $\Gamma$ is void the rule $(\rightarrow \square)$ is called the rule of tautology, abbreviated as (RT), after G. H. von Wright [6]. Now we put the following restriction about the rule $(\rightarrow \square)$ :

"Restriction": In one and the same string of an S2*-proof-figure under an $(R T)$ there never appears a rule $(\rightarrow \square)$.

\section{§2. Cut-elimination theorem ${ }^{3)}$ for $S 2^{*}$.}

Theorem. Any S2*-proof-figure can be transformed into an S2*. proof-figure with the same endsequent and without any cut as a rule of inference.

Proof. As usual we replace all cut-rules by mix-rules and consider the case when a mix-rule appears in the lowest part only; the definition of grade and rank of a mix being the same as Gentzen gives, the proof will be carried out by induction on grade and rank.

When the outer-most (logical) symbol of the mix-formula is not $\square$, it is almost clear that we can decrease the rank or the grade of the mix by using Gentzen's transformations with slight modifications. So we have only to consider the case when the mix-formula is of the form $\square \alpha$. Moreover when either of the two upper rules of the mix in question is an $L K$-rule, we can again decrease the rank of the mix by Gentzen's transformations. Now let us consider the following three cases:

Case 1. The left upper rule is a $(\square \rightarrow)$-rule. The lowest part of the proof-figure is :

$$
\frac{\frac{\alpha, \Gamma \rightarrow \Theta}{\square \alpha, \Gamma \rightarrow \Theta}(\square \rightarrow) \quad \Sigma \rightarrow \mathrm{II}}{\square \alpha, \Gamma, \Sigma_{\square \beta} \rightarrow \Theta_{\square \beta}, \mathrm{II}}(\square \beta) ;
$$

where $(\square \beta)$ denotes that the mix-formula is $\square \beta$, and $\Sigma_{\square \beta}, \Theta_{\square \beta}$ etc. mean the series which arise when we remove all $\square \beta$ 's from $\Sigma$, $\Theta$ etc.

We transform as follows:

$$
\frac{\alpha, \Gamma \rightarrow \Theta \quad \Sigma_{\rightarrow} \rightarrow \mathrm{II}}{\frac{\alpha, \Gamma, \Sigma_{\square \beta} \rightarrow \Theta_{\square \beta}, \mathrm{II}}{\square \beta}(\square)}(\square) ;
$$

3) Gentzen's Hauptsatz. See [1]. 
where the left rank of the new mix is decreased by 1 . Hence we can eliminate this mix by the assumption of induction.

Case 2. The left upper rule is a $(\rightarrow \square)$-rule and the right upper rule is a $(\square \rightarrow)$-rule. The lowest part is :

$$
\frac{\frac{\Gamma \rightarrow \alpha}{\square \Gamma \rightarrow \square \alpha}(\rightarrow \square) \quad \frac{\beta, \Sigma \rightarrow \Pi}{\square \beta, \Sigma \rightarrow \Pi}(\square \rightarrow)}{\square \Gamma,(\square \beta)_{\square \alpha}, \Sigma_{\square \alpha} \rightarrow \Pi}(\square \alpha) ;
$$

where $(\square \beta)_{\square \alpha}$ denotes an empty place when $\alpha$ equals $\beta$, and $\square \beta$ otherwise.

Subcase 2.1. $\Sigma$ does not contain $\square \alpha$, hence $\alpha$ equals $\beta$. We transform as follows :

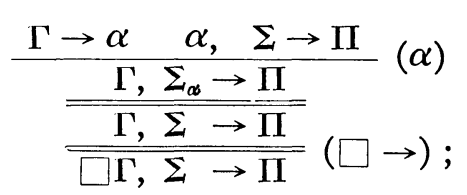

where the grade of the new mix is decreased by 1 . Hence we can eliminate it.

Subcase 2.2. $\Sigma$ contains $\square \alpha$.

Subcase 2.21. $\alpha$ equals $\beta$. We transform as follows:

$$
\begin{aligned}
& \frac{\square \Gamma \rightarrow \square \alpha \quad \alpha, \Sigma \rightarrow \Pi}{\square \Gamma, \alpha, \Sigma_{\square,} \rightarrow \Pi}(\square \alpha)
\end{aligned}
$$

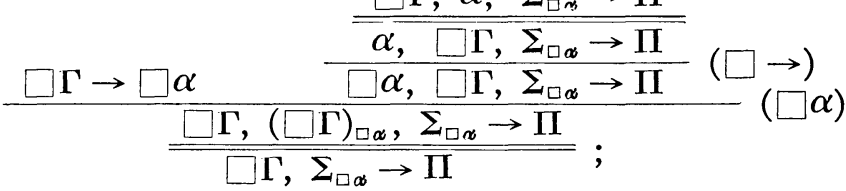

where the right rank of the upper mix is decreased by 1 , and the right rank of the lower mix is 1 except the straightforward case when $\square \Gamma$ contains $\square \alpha$. Hence we can eliminate both of them.

Subcase 2.22. $\alpha$ differs from $\beta$. We transform as follows :

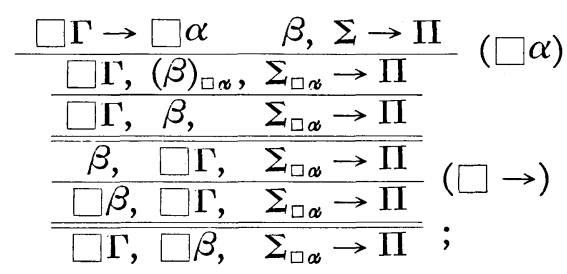

where the right rank of the new mix is decreased by 1 . Hence we can eliminate it. 
Case 3. Both of the upper rules of the mix are $(\rightarrow \square)$-rules. The lowest part is :

$$
\frac{\frac{\Gamma \rightarrow \alpha}{\square \Gamma \rightarrow \square \alpha}(\rightarrow \square) \quad \frac{\Sigma \rightarrow \beta}{\square \Sigma \rightarrow \square \beta}(\rightarrow \square)}{\square \Gamma,(\square \Sigma)_{\square \alpha} \rightarrow \square \beta}(\square \alpha) .
$$

We transform as follows:

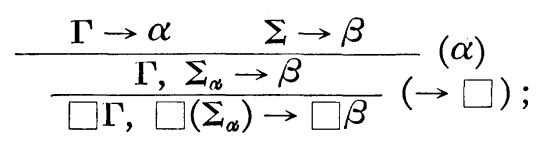

where the grade of the new mix is decreased by 1 . Hence we can eliminate it. Since the upper part of $\Gamma \rightarrow \alpha$ as well as that of $\Sigma \rightarrow \beta$ did not contain any (RT) by the "Restriction", we are able to use the ( $\rightarrow \square$ )rule. And $\square\left(\Sigma_{\infty}\right)$ and $(\square \Sigma)_{\square \infty}$ are clearly identical.

Thus the proof of our theorem is complete.

\section{§. Equivalence of $S 2$ and $S 2 *$.}

3.1. Theorem. If a formula $\alpha$ is provable in S2, then the sequent $\rightarrow \alpha$ is provable in S2*.

Proof. In case $\alpha$ is the axiom $\left(1^{\prime}\right)$,

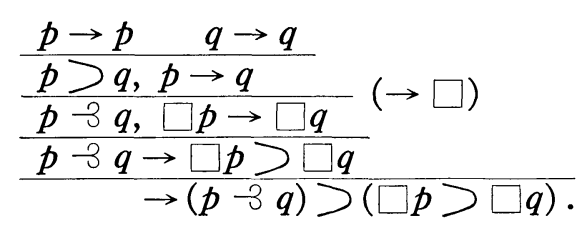

In case $\alpha$ is the axiom (2),

$$
\frac{\frac{p \rightarrow p}{\square p \rightarrow p}(\square \rightarrow)}{\rightarrow \square p>p}
$$

As to the rule $\left(a^{\prime}\right):$ In case $\alpha$ is a tautology, the sequent $\rightarrow \alpha$ being provable in LK, we can use (RT) and $\rightarrow \square \alpha$ is provable in S2*. Also in case $\alpha$ is one of the additional axioms $\left(1^{\prime}\right)$ and (2), $\rightarrow \alpha$ is provable without (RT) as is seen above, hence $\rightarrow \square \alpha$ is provable by (RT).

As to the rule (b) we shall first prove the following

3.2. Lemma. If a sequent $\rightarrow \square \alpha$ is provable in $S 2^{*}$, then the sequent $\rightarrow \alpha$ is provable in E2*, where E2* is the sequential calculus which arises when we remove the $(R T)$ from $S 2^{*}$. In other words $\rightarrow \alpha$ is provable without $(R T)$. 
Proof. Since the cut-elimination theorem holds for $S 2^{*}$, we may assume that the sequent $\rightarrow \square \alpha$ is proved without any cut. Eliminating one after another all formulas $\square \alpha$ 's appearing in the succedents of sequents of this proof-figure, we can no more get a correct $S 2^{*}$-prooffigure. For otherwise, the sequent $\rightarrow$ would be provable in S2*, which is surely impossible.

Therefore there exists at least one rule of inference which does not hold in $S 2^{*}$ after the elimination of $\square \alpha$ 's. In other words the following form must appear at least once in the given proof-figure:

$$
\frac{\Gamma \rightarrow \alpha}{\square \Gamma \rightarrow \square \alpha}(\rightarrow \square)
$$

Supposing here $\Gamma$ is not empty, we see that all formulas of $\square \Gamma$ should be subformulas of $\square \alpha$ which is the only formula appearing in the endsequent. But this is impossible. For there is no cut at all and $(\rightarrow \square)$ rule has only one formula in both its succedents. Accordingly $\mathbf{\Gamma}$ must be empty, and the sequent $\rightarrow \alpha$ is surely provable. Moreover since the upper part of $\Gamma \rightarrow \alpha$ did not contain any (RT), $\rightarrow \alpha$ is proved without (RT).

Now we shall consider the rule (b) of $S 2$. If $\alpha-3 \beta$ is proved in $S 2^{*}$, then by the above Lemma the sequent $\rightarrow \alpha \supset \beta$ is provable without (RT). Therefore we proceed as follows:

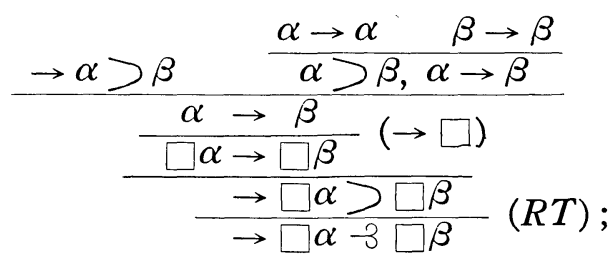

where the "restriction" is surely satisfied.

This completes the proof of Theorem 3.1.

3.3. Theorem. If a sequent $\rightarrow \alpha$ is provable in $\mathrm{S2}^{*}$, then the formula $\alpha$ is provable in $\mathrm{S} 2$.

Proof. We shall prove more generally that if the sequents $\alpha_{1}, \alpha_{2}, \cdots$, $\alpha_{m} \rightarrow \beta_{1}, \beta_{2}, \cdots, \beta_{n} ; \rightarrow \beta_{1}, \beta_{2}, \cdots, \beta_{n} ; \alpha_{1}, \alpha_{2}, \cdots, \alpha_{m} \rightarrow$ are provable in $S 2^{*}$ then the formulas $\left(\alpha_{1} \& \alpha_{2} \& \ldots \& \alpha_{m}\right) \supset\left(\beta_{1} \vee \ldots \vee \beta_{n}\right) ; \beta_{1} \vee \beta_{2} \vee \ldots \vee \beta_{n}$; $\sim\left(\alpha_{1} \& \alpha_{2} \& \cdots \& \alpha_{m}\right)$ are respectively provable in $S 2$. We shall call these formulas the "interpretations" of the corresponding sequents.

Now in the formulation of $S 2$ we replace the rule (b) by the following rule

(Eb): if $\alpha \supset \beta$ is provable, then so is $\square \alpha \supset \square \beta$.

The system which then arises is called E2 after Lemmon [2]. 
3. 4. Lemma. If a sequent $\Gamma \rightarrow \Theta$ is provable in E2*, then its interpretation is provable in $E 2^{4}$.

Proof. We have only to prove the formula $(\square p \& \square q)>\square(p \& q)$ in E2. We have $p \supset(q>(p \& q))$. Then by (Eb) $\square p \supset(q-3(p \& q))$. From $\left(1^{\prime}\right)$ we have $(q-3(p \& q)) \supset(\square q \supset \square(p \& q))$. Hence $\square p \supset(\square q$ $\supset \square(p \& q)$ ), accordingly $(\square p \& \square q) \supset \square(p \& q)$ holds in E2.

Now we return to the proof of Theorem 3.3. Of all the rules of $S 2^{*}$ except only $(\rightarrow \square)$-rule it is clear that from the interpretation(s) of the upper sequent(s) we can deduce the interpretation of the lower sequent. As to $(\rightarrow \square)$-rule :

$$
\frac{\Gamma \rightarrow \alpha}{\square \Gamma \rightarrow \square \alpha}(\rightarrow \square)
$$

its upper sequent $\Gamma \rightarrow \alpha$ is provable in $E 2^{*}$ by the "restriction", hence its interpretation is provable in E2 by Lemma 3.4. On the other hand, it is almost clear that for an E2-provable formula $\alpha$ the formula $\square \alpha$ is provable in $S 2^{5)}$. So when $\Gamma$ is empty, $\alpha$ is provable in $E 2$, hence $\square \alpha$ is provable in S2. And when $\Gamma$ is not empty, the lower sequent $\square \Gamma \rightarrow \square \alpha$ is proved in $E 2^{*}$, hence its interpretation holds in $E 2$, a fortiori in $S 2$. This completes the proof of Theorem 3.3.

Thus we have gained a direct Gentzen decision procedure for Lewis's system $S 2$.

\section{§4. Formulations of $S 3$ and $53 *$.}

Lewis's system $S 3$ is, also after Lemmon [2], the system which arises when in the formulation of $S 2$ we replace the axiom $\left(1^{\prime}\right)$ by

$$
(p-3 q)>(\square p-3 \square q) \text {, }
$$

and we remove the rule (b) (which becomes redundant from the axiom (1)).

Now we shall present a sequential calculus $S 3^{*}$, which arises when in the formulation of $S 2^{*}$ we replace the rule $(\rightarrow \square)$ by the following

$$
\frac{\Gamma \rightarrow \alpha, \square \Theta \quad \square \Gamma \rightarrow \alpha}{\square \Gamma \rightarrow \square \alpha}(\rightarrow \square)
$$

4) Cleary for an E2-provable formula $\alpha$ the sequent $\rightarrow \alpha$ is provable in $E 2^{*}$. Therefore E2 and $E 2^{*}$ are equivalent.

5) The converse holds also true, by Theorem 3.1., Lemma 3.2., and Lemma 3.4. Therefore E2 provides all theses of the form $\square x$ of $S 2$ in "material" form. 
In case $\Gamma$ is empty, we shall call it (RT) as well $1^{6}$.

"Restrictions" for the rule $(\rightarrow \square):\left(1^{\circ}-3^{\circ}\right)$

$1^{\circ}$ Each formula of $\square \Theta$ is a subformula of $\alpha$ or of a formula belonging to $\Gamma$. $(\square \Theta \text { may be emtpy })^{7}$.

$2^{\circ} \quad$ The upper part of the sequent $\Gamma \rightarrow \alpha, \square \Theta$ contains LK-rules only. (Of course modal formulas may appear.)

$3^{\circ} \quad$ In one and the same string of an S3*-proof-figure under an $(R T)$ there never appears a rule $(\rightarrow \square)$.

\section{$\S 5$. Cut-elimination theorem for $S^{*}$.}

5.1. Theorem. Any S3*-proof-figure can be transformed into an S3*-proof-figure with the same endsequent and without any cut as a rule of inference.

Proof. The proof runs at first in almost the same way as for $S 2^{*}$. Namely Case 1 . is just the same.

Case 2.

$$
\frac{\Gamma \rightarrow \alpha, \square \Theta \quad \square \Gamma \rightarrow \alpha}{\square \Gamma \rightarrow \square \alpha}(\rightarrow \square) \quad \frac{\beta, \Sigma \rightarrow \Pi}{\square \beta, \Sigma \rightarrow \Pi}(\square \rightarrow)
$$

Subcase 2.1. $\Sigma$ does not contain $\square \alpha$, hence $\alpha$ equals $\beta$. We transform as follows:

$$
\frac{\square \Gamma \rightarrow \alpha \quad \alpha, \Sigma \rightarrow \Pi}{\frac{\square \Gamma, \Sigma_{\alpha} \rightarrow \Pi}{\square \Gamma, \Sigma \rightarrow \Pi}}(\alpha)
$$

where the grade of the new mix is decreased by 1 .

Subcase 2.2. $\Sigma$ contains $\square \alpha$. We transform in just the same way as for $S 2^{*}$.

Case 3.

$$
\left.\frac{\Gamma \rightarrow \alpha, \square \Theta \quad \square \Gamma \rightarrow \alpha}{\square \Gamma \rightarrow \square \alpha}(\rightarrow \square) \quad \frac{\Sigma \rightarrow \beta, \square \Pi \quad \square \Sigma \rightarrow \beta}{\square \Sigma \rightarrow \square \beta}(\square \alpha) . \square\right)
$$

6) Of course (RT) of $S 3^{*}$ is of different form from (RT) of $S 2^{*}$. But these two rules are deductively equivalent. Clearly (RT) of $S 2^{*}$ is not of weaker form than (RT) of $S 3^{*}$. On the other hand if a sequent $\rightarrow \alpha$ is provable without (RT), then with appropriate additional $\square \Theta$ the sequent $\rightarrow \alpha, \square \Theta$ becomes LK-provable, because the lower sequent $\square \alpha, \Gamma \rightarrow \Theta$ of $(\square \rightarrow)$ rule becomes LK-provable when added $\square \alpha$ to its succedent, and also the lower sequent $\square \mathrm{r}$ $\rightarrow \square \alpha$ of ( $\rightarrow \square$ )-rule (except when $\Gamma$ is empty) becomes LK-provable when added $\square \mathrm{r}$ to its succedent.

7) This rule enjoys the so-called sub-formula property contrary to the $(\rightarrow \square)$-rule which Matsumoto made use of in [4]. 
First we must prove the following

5.2. Lemma. If a sequent of the form $\Gamma \rightarrow \alpha, \square \Theta$ is provable with LK-rules only, then we can eliminate those formulas of $\square \Theta$ which are neither subformulas of $\alpha$ nor subformulas of a formula belonging to $\Gamma$, and leave the remaining sequent also provable with LK-rules only.

Proof. Of course we may assume that $\Gamma \rightarrow \alpha, \square \Theta$ is proved without any cut. If in this proof-figure we eliminate a formula belonging to $\square \Theta$ everywhere in the succedents from the bottom one after another and if still we get a correct LK-proof-figure, then this formula is found redundant and eliminable in the endsequent. If on the contrary when we eliminate a formula $\square \theta$ belonging to $\square \Theta$ everywhere and if the remaining figure no more rests as a correct LK-proof-figure, then the formula $\square \theta$ should have appeared in a beginning sequent $\square \theta \rightarrow \square \theta$; the $\square \theta$ in the antecedent of this sequent must be a subformula of $\alpha$ or of a formula belonging to $\Gamma$, since there is no cut at all.

Now let us consider the Case 3. First we mix the following two sequents :

$$
\frac{\Gamma \rightarrow \alpha, \square \Theta \quad \Sigma \rightarrow \beta, \square \Pi}{\Gamma, \Sigma_{\alpha} \rightarrow(\square \Theta)_{\alpha}, \beta, \square \Pi}(\alpha)
$$

where the lower sequent being proved with LK-rules only, it is provable without cut. Moreover the above Lemma 5.2. assures that we can transform this proof-figure until it has the endsequent $\Gamma, \Sigma_{\alpha} \rightarrow \beta, \square \Xi$ where each formula of $\square \equiv$ is a subformula of $\beta$ or of a formula belonging to $\Gamma$ or to $\Sigma_{\infty}$.

Subcase 3.1. $\Gamma$ is not empty. We transform as follows:

$$
\frac{\Gamma, \Sigma_{\alpha} \rightarrow \beta, \square \equiv \quad \frac{\square \Gamma \rightarrow \square \alpha \quad \square \Sigma \rightarrow \beta}{\square \Gamma,(\square \Sigma)_{\square \alpha} \rightarrow \beta}(\square \alpha)}{\square \Gamma,(\square \Sigma)_{\square \alpha} \rightarrow \square \beta}(\rightarrow \square) ;{ }^{8)}
$$

where the right rank of the new mix is decreased by 1 , hence it is eliminable. Since $\Gamma$ is not empty, there is no (RT) at all in the upper part of the above $(\rightarrow \square)$-rule, hence it is admissible. Thus we get the desired endsequent without any mix.

Now we have the following

5. 3. Subtheorem. When a sequent is proved in $S 3^{*}$ without any $(R T)$, then it is provable without any cut, too. In other words, Cut-elimination

8) $\square\left(\Sigma_{\alpha}\right)$ and $(\square \Sigma)_{\square \alpha}$ are identical. 
Theorem holds true for the subsystem E3*, which arises from S3* by the prohibition of the use of $(R T)$.

Subcase 3.2. $\Gamma$ is empty. The lowest part in question is:

$$
\frac{\rightarrow \alpha, \square \Theta \quad \rightarrow \alpha}{\rightarrow \square \alpha}(R T) \quad \frac{\Sigma \rightarrow \beta, \square \Pi \quad \square \Sigma \rightarrow \beta}{\square \Sigma \rightarrow \square \beta}(\square \alpha) .
$$

In this case we are unable to transform in the same way as before, because the sequent $\rightarrow \square \alpha$ being proved with (RT), the "restrictions" for $(\rightarrow \square)$ are no more satisfied after the transformation.

Subcase 3.21. $\square \Xi$ is empty. We transform as follows:

$$
\frac{\Sigma_{\alpha} \rightarrow \beta \quad \frac{\Sigma_{\alpha} \rightarrow \beta}{\overline{\square\left(\Sigma_{\alpha}\right) \rightarrow \beta}}(\square \rightarrow)}{\square\left(\overline{\left.\Sigma_{\alpha}\right) \rightarrow \square \beta}(\rightarrow \square) ;{ }^{8)}\right.}
$$

where all "restrictions" for $(\rightarrow \square)$-rule are surely satisfied.

Subcase 3.22. (The last case). $\square \Xi$ is not empty; let it be $\square \xi_{1}, \cdots$, $\square \xi_{n}(n \geqq 1)$. The following rules

$$
\frac{\frac{\rightarrow \alpha, \square \Theta}{\xi_{i} \rightarrow \alpha, \square \Theta} \quad \frac{\rightarrow \alpha}{\square \xi_{i} \rightarrow \alpha}}{\square \xi_{i} \rightarrow \square \alpha}(\rightarrow \square)
$$

(for $i=1,2, \cdots, n$ ) are clearly all admissible. Mixing the sequents $\Sigma_{\alpha} \rightarrow$ $\beta, \square \Xi$ and $\square \xi_{i} \rightarrow \square \alpha(i=1,2, \cdots, n)$ one after another, and contracting $\square \alpha$ 's in the succedent, we get finally the sequent $\Sigma_{\alpha} \rightarrow \beta, \square \alpha$. Then we proceed as follows :

$$
\frac{\frac{\Sigma_{\alpha} \rightarrow \beta, \square \alpha \quad \square \Sigma \rightarrow \beta}{\frac{\Sigma_{\alpha},(\square \Sigma)_{\square \alpha} \rightarrow \beta, \beta}{(\square \alpha)}}(\square \alpha)}{\left.\frac{\frac{\Sigma_{\alpha},(\square \Sigma)_{\square \alpha} \rightarrow \beta}{\square\left(\Sigma_{\gamma}\right),(\square \Sigma)_{\square \alpha} \rightarrow \beta}}{\frac{(\square \Sigma)_{\square \alpha} \rightarrow \beta}{(\square)}} ; \rightarrow\right)}
$$

Thus we have proved the sequent $(\square \Sigma)_{\square \infty} \rightarrow \beta$ in $E 3^{*}$, hence we can apply the above Subtheorem 5.3., and so it is provable without any cut. Accordingly

$$
\frac{\Sigma_{\alpha} \rightarrow \beta, \square \Xi \quad(\square \Sigma)_{\square \alpha} \rightarrow \beta}{(\square \Sigma)_{\square \alpha} \rightarrow \square \beta}(\rightarrow \square)
$$

becomes admissible.

This completes the proof of Theorem 5.1. 
§. Equivalence of $S 3$ and $S 3^{* 9}$.

6.1. Theorem. If a formula $\alpha$ is provable ih S3, then the sequent $\rightarrow \alpha$ is provable in S3*.

Proof. When $\alpha$ is the axiom (1),

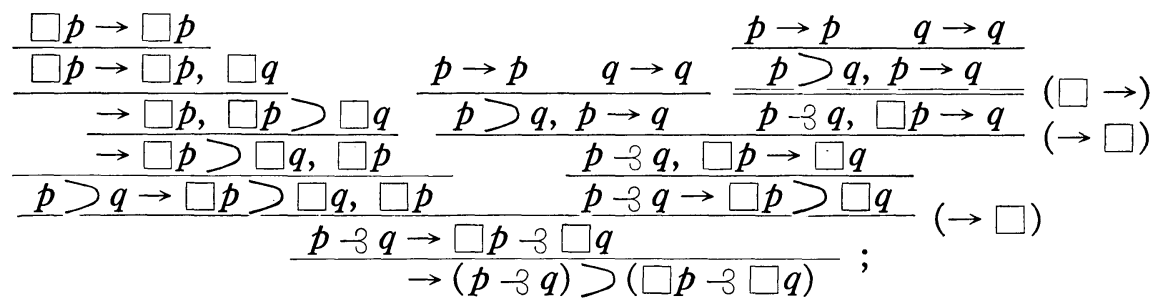

where "restrictions" are certainly satisfied for both $(\rightarrow \square)$-rules.

When $\alpha$ is the axiom (2), $\rightarrow \alpha$ is clearly provable.

As to the rule $\left(a^{\prime}\right)^{10)}$ : in case $\alpha$ is a tautology $\rightarrow \alpha$ is provable in LK, hence we can apply (RT) and $\rightarrow \square \alpha$ becomes provable. In case $\alpha$ is the axiom (1)

$$
\begin{aligned}
& \frac{p-3 q}{p-3 q} \rightarrow p-3 q \\
& \rightarrow(p-3 q)>(\square p-3 \square q), p-3 q \rightarrow(p-3 q)>(\square p-3 \square q) \\
& \rightarrow(p<q)-3(\square p-3 \square q) .
\end{aligned}
$$

In case $\alpha$ is the axiom (2)

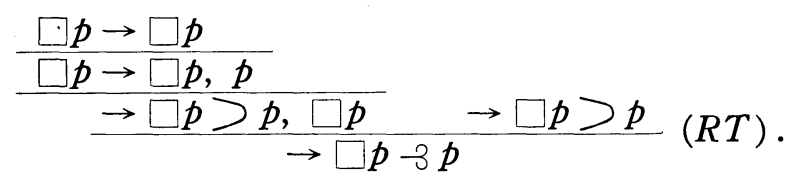

This completes the proof of theorem 6.1.

6. 2. Theorem. If a sequent $\rightarrow \alpha$ is provable in $\mathrm{S}^{*}$, then the formula $\alpha$ is provable in S3.

Proof. We shall prove more generally that for any rule of $S 3^{*}$ from the interpretation(s) of its upper sequent(s) we can deduce in S3 the interpretation of its lower sequent. To show this we have only to consider the $(\rightarrow \square)$-rule. Now we need the following

6.3. Lemma. When a sequent $\Gamma \rightarrow \Theta$ is provable in E3*, it is also

9) Contrary to the case of the equivalence of $S 2$ and $S 2^{*}$ we need not here the cutelimination theorem for $S 3^{*}$.

10) Cf. footnote 6). 


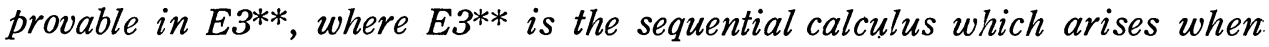
in E2* the use of the sequent $\alpha-3 \beta \rightarrow \square \alpha-3 \square \beta$ as a bzginning sequent is admitted ${ }^{11122}$.

Proof. We have only to show that the $(\rightarrow \square)$-rule of $E 3^{*}$ is admissible in $E 3^{* *}$. We put $\Gamma$ as $\gamma_{1}, \cdots, \gamma_{n}$ and $\Theta$ as $\theta_{1}, \cdots, \theta_{m}(n \geqq 1, m \geqq 0)$.

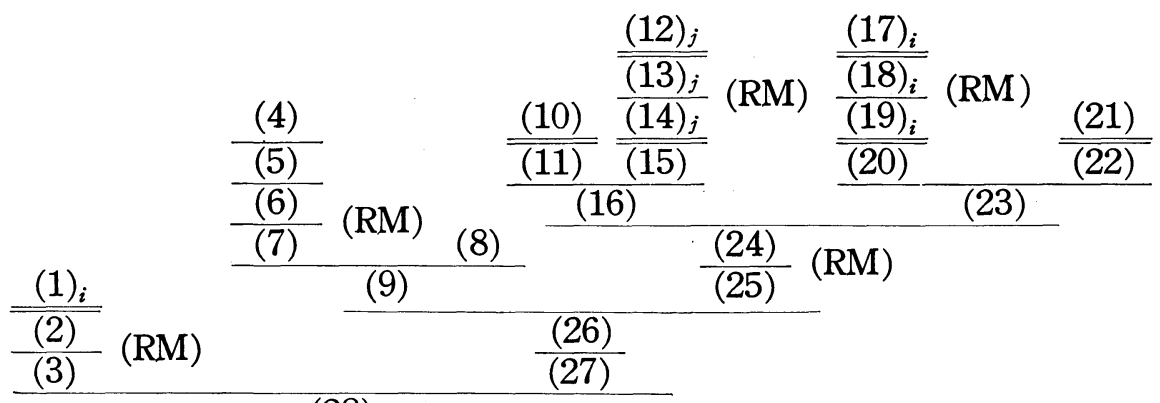

(28)

Here $(\mathrm{RM})^{13)}$ denotes the $(\rightarrow \square)$-rule of $E 2^{*}$, and numbers in brackets denote the following sequents respectively:

$$
\begin{gathered}
\gamma_{i} \rightarrow \gamma_{i} \quad(i=1, \cdots, n) . \\
\gamma_{1}, \cdots, \gamma_{n} \rightarrow \gamma_{1} \& \cdots \& \gamma_{n} . \\
\square \gamma_{1}, \cdots, \square \gamma_{n} \rightarrow \square\left(\gamma_{1} \& \cdots \& \gamma_{n}\right) . \\
\gamma_{1} \& \cdots \& \gamma_{n} \rightarrow \gamma_{1} \& \cdots \& \gamma_{n} .
\end{gathered}
$$$$
\gamma_{1} \& \cdots \& \gamma_{n}, \theta_{1} \vee \cdots \vee \theta_{m} \rightarrow \gamma_{1} \& \cdots \& \gamma_{n} .
$$$$
\gamma_{1} \& \cdots \& \gamma_{n} \rightarrow\left(\theta_{1} \vee \ldots \vee \theta_{m}\right)>\left(\gamma_{1} \& \cdots \& \gamma_{n}\right) \text {. }
$$$$
\square\left(\gamma_{1} \& \cdots \& \gamma_{n}\right) \rightarrow\left(\theta_{1} \vee \ldots \vee \theta_{m}\right)-3\left(\gamma_{1} \& \cdots \& \gamma_{n}\right) .
$$$$
\left(\theta_{1} \vee \ldots \vee \theta_{m}\right)-3\left(\gamma_{1} \& \cdots \& \gamma_{n}\right) \rightarrow \square\left(\theta_{1} \vee \ldots \vee \theta_{m}\right)-\square\left(\gamma_{1} \& \cdots \& \gamma_{n}\right)
$$

(beginning sequent).

11) Therefore $E 3^{* *}$ and $Q^{*}$ in [4] are identical.

12) It is almost clear that the converse of Lemma 6.3. holds. (See the proof of Theorem 6.1.) So $E 3^{*}$ and $E 3^{* *}$ are equivalent.

13) Abbreviation of "rule of monotonicity", as we might call it. 


$$
\begin{gathered}
\square \vee \theta_{1} \cdots \vee \square \theta_{m} \rightarrow \square\left(\theta_{1} \vee \ldots \vee \theta_{m}\right) . \\
\gamma_{1} \& \cdots \& \gamma_{n} \rightarrow \alpha, \square\left(\theta_{1} \vee \cdots \vee \theta_{m}\right) . \\
\gamma_{i} \rightarrow \gamma_{i} \quad(i=1, \cdots, n) . \\
\gamma_{1} \& \cdots \& \gamma_{n} \rightarrow \gamma_{i} \quad(i=1, \cdots, n) . \\
\square\left(\gamma_{1} \& \cdots \& \gamma_{n}\right) \rightarrow \square \gamma_{i} \quad(i=1, \cdots, n) . \\
\square\left(\gamma_{1} \& \cdots \& \gamma_{n}\right) \rightarrow \square \gamma_{1} \& \cdots \& \square \gamma_{n} . \\
\square \gamma_{1}, \cdots, \square \gamma_{n} \rightarrow \alpha \quad(p r e m i s e) . \\
\square \gamma_{1} \& \cdots \& \square \gamma_{n} \rightarrow \alpha . \\
\square\left(\gamma_{1} \& \cdots \& \gamma_{n}\right) \rightarrow \alpha . \\
\square\left(\gamma_{1} \& \cdots \& \gamma_{n}\right), \square\left(\theta_{1} \vee \cdots \vee \theta_{m}\right)-\square\left(\gamma_{1} \& \cdots \& \gamma_{n}\right) \rightarrow \square \alpha . \\
\square\left(\gamma_{1} \& \cdots \& \gamma_{n}\right), \square\left(\gamma_{1} \& \cdots \& \gamma_{n}\right) \rightarrow \square \alpha . \\
\square\left(\gamma_{1} \& \cdots \& \gamma_{n}\right) \rightarrow \square \alpha . \\
\square \gamma_{1}, \cdots, \square \gamma_{n} \rightarrow \square \alpha .
\end{gathered}
$$

(28) is the sequent to be required.

6.4. Lemma. If a sequent is provable in E3**, then its interpretation is provable in E3, where E3 is the system which arises when in E2 the axiom $\left(1^{\prime}\right)$ is replaced with $(1)^{1415)}$.

Proof. Clear.

Now let us return to the proof of our Theorem 6.2. When $\Gamma$ is empty in the $(\rightarrow \square)$-rule of $S 3^{*}, \rightarrow \alpha$ is provable in $E 3^{*}$, accordingly by the Lemmas 6.3. and 6.4. the formula $\alpha$ is provable in E3. On the other hand, it is almost clear that for an E3-provable formula $\alpha$ the formula $\square \alpha$ is provable in $S 3^{16)}$. And when $\Gamma$ is not empty, the lower sequent $\square \Gamma \rightarrow \square \alpha$ is proved in $E 3^{*}$, hence its interpretation holds in $E 3$, a fortiori in S3. This completes the proof of Theorem 6.2.

Thus we have obtained a direct Gentzen decision procedure for Lewis's system S3.

(Received February 27, 1961)

14) $E 3$ is also described in [2].

15) Clearly the converse of this Lemma holds true, hence $E 3$ and $E 3^{*}$ are equivalent. (See footnote 12)).

16) Conversely if $\square \alpha$ is provable in $S 3$, then $\rightarrow \square \alpha$ is provable in $S 3^{*}$, and using similar tricks as in the proof of Lemma 3.2. we can conclude that the sequent $\rightarrow \alpha$ is already proved in $E 3^{*}$, hence $\alpha$ is provable in $E 3$. Therefore $E 3$ provides all theses of the form $\square \alpha$ of $S 3$ in "material" form. 


\section{Bibliography}

[1] G. Gentzen: Untersuchungen über das logische Schliessen, I. II., Math. Z. 39 (1935), 176-210, 405-431.

[2] E. J. Lemmon: New foundations for Lewis modal systems, J. Symb. Logic 22 (1957), 176-186.

[3] C. I. Lewis and C. H. Langford: Symbolic logic, New York (1932).

[4] K. Matsumoto: Decision procedure for modal sentential calculus S3, Osaka Math. J. 12 (1960), 167-175.

[5] M. Ohnishi and K. Matsumoto: Gentzen method in modal calculi, Osaka Math. J. 9 (1957), 113-130.

[6] G. H. von Wright: An essay in modal logic, Amsterdam (1951). 
